\title{
The Shaping of Modern Ireland - A Centenary Assessment d' Eugenio Biagini
}

\section{Karin Fischer}

\section{OpenEdition}

1 Journals

\section{Édition électronique}

URL : http://journals.openedition.org/etudesirlandaises/5061

DOI : 10.4000/etudesirlandaises.5061

ISSN : 2259-8863

\section{Éditeur}

Presses universitaires de Caen

\section{Édition imprimée}

Date de publication : 30 novembre 2016

Pagination : 192-193

ISBN : 978-2-7535-5358-3

ISSN : 0183-973X

\section{Référence électronique}

Karin Fischer, "The Shaping of Modern Ireland - A Centenary Assessment d' Eugenio Biagini », Études irlandaises [En ligne], 41-2 | 2016, mis en ligne le 30 janvier 2016, consulté le 24 septembre 2020. URL: http://journals.openedition.org/etudesirlandaises/5061; DOI : https://doi.org/10.4000/ etudesirlandaises.5061

\section{(c) (†)(อ)}

Études irlandaises est mise à disposition selon les termes de la Licence Creative Commons Attribution - Pas d'Utilisation Commerciale - Partage dans les Mêmes Conditions 4.0 International. 
duelle »; elle se reflète aussi dans le texte par le recours à l'allusion. Au contraire de la citation ou de la référence qui sont lisibles et franches, cet " emprunt non littéral » et «non explicite » qu'est l'allusion ajoute insidieusement à l'impression d'une désintégration de l'univers de McCann, car elle "suppose un détour, une déviation, un décrochage du texte lu » pour le lecteur.

Bertrand Cardin en vient ainsi à étudier le concept d'influence, qui " repose étymologiquement sur l'image de l'écoulement d'un flux » et suppose donc une certaine linéarité chronologique, ou une ascendance. S'il soutient l'idée que l'écriture de McCann est " hantée par la nostalgie d'une origine ", il souligne pourtant que l'intertexte est " une action réciproque et une interaction mutuelle » et pointe donc la qualité plus rhizomique qu'arborescente d'un texte qui en convoque d'autres. Le pouvoir de l'auteur vient donc à être questionné, dans la mesure où l'intertextualité implique une "coprésence " des textes, une "dilution" et un " décentrement ", plutôt que leur organisation stratifiée.

Dans la même logique, Bertrand Cardin réfléchit à la légitimité de vouloir prêter une "intention " à la figure de l'auteur, et cela même si McCann semble s'engager aux côtés des faibles en leur redonnant leur dignité. Dans sa prose, qui infléchit les textes mythiques pour leur donner " une perspective plus matérielle ", les figures religieuses deviennent effectivement des personnages ordinaires voire déchus. Selon Bertrand Cardin, c'est davantage le rôle du lecteur dans la quête de l'intertexte ou de l'intratexte qu'il faut considérer. Les textes de McCann, qui sont de véritables écrins dans lesquels reposent ceux d'illustres prédécesseurs, « donnent libre cours à une créativité renouvelée "dont, indéniablement, celle du lecteur dont le monde est " enrichi par l'Autre » au contact des textes.

Cécile MAudet

Eugenio Biagini, Daniel Mulhal (eds), The Shaping of Modern Ireland - A Centenary Assessment, Dublin, Irish Academic Press, 2016, xxI + 248 p., ISBN 978-1-911024-00-2.

Eugenio Biagini, Professeur d'Histoire moderne et contemporaine à l'université de Cambridge, et Daniel Mulhall, diplomate de carrière, actuel ambassadeur d'Irlande en Grande-Bretagne, ont eu l'idée de s'inspirer directement de l'ouvrage The Shaping of Modern Ireland qui avait été publié en 1960 sous la direction de Conor Cruise O'Brien, pour en proposer une forme de relecture historiographique collective dans le contexte actuel de la " décennie des commémorations ». Louvrage dirigé par Cruise O'Brien revenait sur le tournant du vingtième siècle 
en Irlande par le prisme des histoires individuelles de figures considérées comme ayant contribué à "façonner " l'Irlande moderne de manière significative. Pour mener à bien leur entreprise, Biagini et Mulhall ont fait appel à une palette impressionnante d'historiens irlandais, de Joseph Lee à Diarmaid Ferriter en passant par Mary E. Daly et Vincent Comerford notamment, mais aussi à des acteurs de la scène politique et artistique irlandaise contemporaine tels que Martin Mansergh et Theo Dorgan. Du point de vue du choix des figures historiques, la différence majeure entre les deux ouvrages, qui reflète une évolution de taille du point de vue des perceptions dominantes de la période comme le font remarquer Biagini et Mulhall, est la place maintenant faite à quelques femmes, qui n'avaient eu aucun droit de cité en 1960. Le nombre de chapitres a ainsi été augmenté pour permettre l'inclusion de Constance Markievicz, Eva Gore-Booth, Hannah Sheehy-Skeffington entre autres. Un chapitre sur des personnages influents du monde économique de l'époque tels que la famille Guinness a également été ajouté.

Les auteurs des différents chapitres proposent une relecture souvent intéressante de l'histoire individuelle de différentes figures historiques (pour la plupart des acteurs de la scène politique irlandaise de l'époque), de leur rapport à l'histoire irlandaise en devenir et de leur influence réelle ou supposée sur les décennies qui ont suivi, et une comparaison systématique avec les chapitres correspondants de l'ouvrage de 1960 peut se révéler passionnante d'un point de vue historiographique (bien que la plupart des auteurs s'inspirent eux-mêmes pour l'essentiel d'autres ouvrages sur les sujets traités).

Dans leur préface Biagini et Mulhall admettent avoir laissé de côté en grande partie la dimension structurelle des changements qui s'opérèrent entre la fin du $\mathrm{XIX}^{\mathrm{e}}$ siècle et le début du $\mathrm{XX}^{\mathrm{e}}$ siècle en Irlande, et notamment les réformes agraires et leur impact, un élément qui manquait déjà dans l'ouvrage dirigé par Cruise O’Brien, comme le fait remarquer Paul Bew dans son chapitre. C'est d'une certaine manière le reproche que l'on pourrait faire plus généralement malgré tout au postulat même de l'ouvrage, à savoir l'idée qu'un nombre restreint d'individus particulièrement influents ou significatifs puissent être réputés avoir "façonné" l'Irlande moderne, au-delà ou en deçà des évolutions structurelles et du jeu des forces et tensions collectives à l'œuvre dans le pays tout entier comme au niveau international. De ce point de vue, l'ouvrage reste relativement académique, voire conservateur, d'un point de vue historiographique toujours, mais les points de vue très divers qui ressortent des différents chapitres, sur l'Irlande d'hier comme sur celle d'aujourd'hui, n'en donnent pas moins amplement matière à réflexion.

Karin FisCHER 